\title{
Avaliação da UFMG Virtual na visão de alunos surdos sob a ótica da Engenharia Semiótica
}

\author{
Letícia Capelão $^{1}$, Erica R. de Oliveira ${ }^{2}$, Reinildes Dias ${ }^{3}$, Elidéa L. A. Bernardino ${ }^{3}$
}

Centro de Apoio à Educação a Distância ${ }^{1}$, Faculdade de Letras ${ }^{3}$

Universidade Federal de Minas Gerais (UFMG) - Belo Horizonte, MG - Brasil

\author{
Centro Universitário Una ${ }^{2}$ - Belo Horizonte, MG - Brasil \\ \{leticiacapelaoacademico, profericaoliveira, reinildes, \\ elideabernardino\} egmail.com
}

\begin{abstract}
The Distance Learning to explore the technology and media is presented as a possible alternative for the access and integration of deaf people in education, especially in higher education. In this work, we sought to identify the main difficulties of using Virtual UFMG Moodle, the deaf public as well as the communication breaks present in the interface. For this, we conducted a case study in the Virtual UFMG Moodle was evaluated from the perspective of deaf students. The results generated indicators that contribute to the accessibility of deaf students in distance education.
\end{abstract}

Resumo. A educação a distância ao explorar a tecnologia e mídias apresentase como uma possível alternativa para o acesso e integração dos surdos no ensino, em especial, no ensino superior. Neste trabalho, buscou-se identificar as principais dificuldades de uso do Moodle da UFMG Virtual, pelo público surdo, bem como as rupturas de comunicação presentes na interface. Para isso, foi realizado um estudo de caso no qual o Moodle da UFMG Virtual foi avaliado sob a perspectiva de alunos surdos. Os resultados geraram indicadores que contribuem com a acessibilidade de alunos surdos na EaD.

\section{Introdução}

A utilização das tecnologias e ambientes virtuais de aprendizagem (AVA) como ferramentas para apoiar a aprendizagem dos alunos é uma realidade em várias instituições de ensino. Esses ambientes possibilitam o compartilhamento de informações, socialização, integração e construção do conhecimento pelos seus usuários. Dentre os AVAs, destacamos o Moodle, por sua grande expansão, sendo o mais utilizado no mundo, em situações de ensino e aprendizagem [Capterra, 2014]. Essa popularização se apresenta como uma possível alternativa para integração do cidadão brasileiro no contexto digital, em especial dos surdos, no ensino superior.

A formação em cursos superiores para os surdos é um grande diferencial que pode possibilitar-lhes o exercício da cidadania, direito à educação e atuação em cargos profissionais de nível superior. Contudo, percebe-se uma grande desatenção às questões de acessibilidade em ambientes digitais e na web para as pessoas surdas [Capelão et al., 2011; Rocha et al., 2014; Pivetta et al., 2013; Capelão, 2015]. Em consequência, os surdos têm sua autonomia e produtividade limitadas ao auxílio de outras pessoas, bem como o seu livre acesso a informação e educação. 
Grande parte das informações e conteúdos didáticos tem sido disponibilizada em uma língua que não é natural aos surdos: a língua portuguesa (LP). Quando se propõe a EaD como um possível caminho para a continuidade de estudo desse público alvo é importante identificar suas necessidades especiais no contexto educacional, assim como as ações a serem adaptadas, em função dos surdos terem a Libras como primeira língua (L1). A avaliação dos AVAs com alunos surdos é uma estratégia que pode contribuir para definir tais parâmetros, a fim de orientar a apresentação de interface destes ambientes, com foco na acessibilidade para esses alunos.

Com o objetivo de contribuir para a pesquisa nesta área apresentamos, neste trabalho, um estudo exploratório que avaliou o AVA Moodle da UFMG, sob a abordagem da Engenharia Semiótica (EngSem) [de Souza, 2005], uma teoria da Interação Humano Computador (IHC). Através desta avaliação foi possível identificar problemas e propor sugestões de melhorias na interface do Moodle da UFMG Virtual, contribuindo, assim, para a acessibilidade de alunos surdos na EaD.

Na seção 2, apresentamos alguns conceitos relacionados aos surdos, Libras e o contexto educacional, e também sobre a EngSem, teoria de IHC que fundamentou este estudo. Na seção 3, apresentamos alguns trabalhos de pesquisa relacionados ao nosso tema. Os resultados da avaliação são apresentados na seção 4, onde abordamos os principais os problemas encontrados e fazemos sugestões de melhorias que podem ser implementadas na interface. Por fim, na seção 5, apresentamos as conclusões e contribuições da avaliação do Moodle da UFMG Virtual de acordo com aplicação do MISI.

\section{Fundamentos Teóricos}

Nesta seção, buscamos caracterizar a Engenharia Semiótica e aspectos relacionados à educação dos surdos e suas relações com a linguagem.

\subsection{Engenharia Semiótica}

A Engenharia Semiótica (EngSem) é uma teoria explicativa de IHC que se concentra na comunicação entre o designer e usuário, sendo feita através da interface de um sistema [de Souza, 2005]. Assim, para avaliar a qualidade de uso da interface a EngSem define a propriedade de comunicabilidade. A comunicabilidade é a capacidade de um sistema comunicar a lógica, a intenção e os princípios de design, de forma organizada e consistente, realizando, assim, sua finalidade junto ao usuário [de Souza, 2005].

Entendemos que, à medida que o aluno surdo utiliza um ambiente de aprendizagem, ele precisa entender como interagir com ele. Assim, nossa avaliação centra-se na comunicabilidade do Moodle da UFMG Virtual, para que possamos contribuir para uma melhor comunicação dos surdos, em ambientes de apoio ao aprendizado.

Para avaliar a comunicabilidade de uma interface a EngSem propõe alguns métodos: Método de Inspeção Semiótica (MIS), Método de Avaliação de Comunicabilidade (MAC) e o MISI (Método de Inspeção Semiótica Intermediado) [de Souza, 2005; de Oliveira, 2010]. Neste trabalho optamos pela utilização do MISI, em virtude de lacunas já apresentadas em pesquisas, também com alunos surdos, utilizando o MIS e MAC [Capelão et al., 2011]. Estes métodos não previam situações peculiares de usuários surdos interagindo em sua L2.

O MISI tem como objetivo entender a comunicação entre o designer e o usuário, mediados por uma interface, num contexto de avaliação de um sistema educacional por 
um usuário indireto (i.e. professor). A inspeção do sistema é intermediada por especialistas em EngSem, através de uma entrevista semiestruturada, que é utilizada durante a inspeção. A aplicação do MISI é apresentada na seção 4. Na seção seguinte, detalhamos aspectos relacionados aos surdos no contexto educacional e suas relações com a Libras e LP.

\subsection{Educação dos surdos e suas relações com a linguagem}

Muitas das pessoas surdas não têm domínio ainda de sua L2 (LP no Brasil), pois não adquiriram e internalizaram a língua de sinais (LS), sua L1, nos primeiros anos de vida. São esses os surdos que enfrentam desafios maiores para adquirir a L2. Isso configura-se como fator chave que dificulta a compreensão dos textos na língua oral (LP), pelos surdos.

Com isso, eles enfrentam graves problemas de comunicação, pois estão inseridos em uma sociedade ouvinte, onde prevalece o uso da língua oral. Assim, eles vivenciam uma situação de exclusão nos níveis social, político e educacional. As variáveis que interferem em seu desenvolvimento e no processo de aprendizagem de sua L1 ainda estão presentes na realidade de muitas crianças, adolescentes e adultos surdos. O desconhecimento sobre a cultura surda e a língua de sinais (LS) é uma delas. Além disso, a lei, que reconhece a LS como meio legal de comunicação e expressão, é recente e o cumprimento da legislação ainda não está ocorrendo em todas as dimensões, especialmente, no âmbito educacional.

Com um olhar para o ensino superior, foco da nossa pesquisa, a inclusão e integração dos surdos é emergente. Dados estatísticos revelam que esse público alvo tem conseguido buscar seu espaço nas instituições universitárias brasileiras nos últimos anos (2013-2014), mas os números ainda são pouco expressivos [Capelão, 2015]. Neste sentido, essa pesquisa contribui à medida que apresenta melhorias que podem ser implementadas em um AVA, a fim de oferecer condições de acesso ao público surdo.

$\mathrm{Na}$ seção seguinte, apresentamos alguns trabalhos que tem sido feito com o intuito de avaliar ambientes educacionais, tanto por ouvintes quanto por surdos.

\section{Trabalhos Relacionados}

No contexto educacional, a qualidade de um sistema traz desafios em sua avaliação: aspectos de aprendizagem pelo aluno, aspectos didáticos e a comunicação proposta pelo professor, através de seu diálogo ou design da aula que também perpassam pela interação dos alunos com o sistema [Preece et al., 2011]. Assim, é relevante investigar se os problemas são relativos às decisões sobre a interface ou ao conteúdo educacional [Prates, 2004]. Apresentamos, nesta seção, algumas pesquisas desenvolvidas com ênfase na avaliação de ambientes educacionais para alunos ouvintes e surdos, que abordam estes aspectos específicos.

Flor et al. [2014] realizaram uma revisão sistemática da literatura buscando identificar as características de projetos de interfaces voltados para o webdesign acessível a surdos. A revisão foi realizada com trabalhos publicados entre os anos 2000 a outubro de 2013, mês em que foi realizada a busca na base de dados. O objetivo do estudo foi diminuir as barreiras de conteúdo e navegação em websites para o público surdo. Como resultado, apresentaram um conjunto das principais recomendações extraídas de estudos que envolvem interface para surdos. 
Rocha et al. [2014] também fizeram uma revisão sistemática da literatura com o objetivo de obter uma visão geral sobre ações para a acessibilidade dos surdos em ambientes virtuais. Os autores concluíram que ainda há uma carência de trabalhos nesta área. Como resultado, criaram um mapa dos estudos mostrando onde os esforços da comunidade têm se concentrado, para criar soluções assistivas voltadas para a educação do surdo em AVAs, como também as técnicas e abordagens utilizadas nesse contexto.

De Oliveira [2010] realizou uma revisão sobre avaliação de ambientes educacionais e uma investigação sobre a aplicabilidade dos métodos de avaliação propostos pela EngSem, como forma de avaliar a comunicabilidade de sistemas educacionais. A autora concluiu que a maioria dos trabalhos utilizam uma combinação de métodos já existentes, capazes de revelar diferentes problemas em sistemas de domínio educacional e, com isso, necessitam de diferentes abordagens para avaliar a interação com o aluno, professor e projetista. Pode-se perceber, ainda, que a maioria dos trabalhos avaliou a usabilidade com foco na aprendizagem, em aspectos educacionais, pedagógicos ou técnicos. Estes resultados evidenciam a carência de pesquisas que contemplem a avaliação de ambientes de apoio ao aprendizado por pessoas surdas, com foco na comunicabilidade de um AVA.

Capelão et al. [2011] avaliou a comunicabilidade da interface do Moodle em LP, na perspectiva de usuários surdos. Os autores avaliaram a comunicabilidade do Moodle da UFMG Virtual, com especialistas e com usuários surdos e ouvintes. Os resultados apresentaram rupturas de comunicação na interface que comprometeram o uso de funcionalidades do Moodle, tanto por alunos surdos, quanto por ouvintes. A partir das rupturas identificadas, observou-se que os problemas de comunicabilidade estavam relacionados à interface e não às dificuldades relacionadas à $\mathrm{LP}$ pelos surdos. $\mathrm{Na}$ aplicação do MAC com usuários surdos foram relatadas situações em que eles sentiram necessidade de tirar dúvidas de contexto linguístico, pela não compreensão de algumas palavras ou expressões em LP e não podiam fazê-lo. Como o MAC é um método que não prevê a intervenção dos pesquisadores, não foi possível aos surdos esclarecer suas dúvidas em relação à LP.

A partir destes resultados e das lacunas evidenciadas nestas pesquisas de acessibilidade e avaliação de comunicabilidade com alunos surdos, propusemos a aplicação do MISI no estudo de caso, descrito na seção seguinte.

\section{Estudo de Caso: aplicação do MISI}

De Oliveira et al. [2008] aplicaram o MISI no contexto de avaliação de um sistema educacional por um usuário indireto (o professor). Em nosso trabalho, aplicamos o MISI no ambiente educacional, com um novo perfil: usuários surdos. O MISI, num contexto de avaliação com usuário surdo, se apresentou com uma potencial contribuição, pois prevê interferências fundamentadas em um método científico, assim como atenção a fatores peculiares deste público. Em se tratando de usuários surdos, em contexto educacional, há que se avaliar tanto a qualidade da interação relacionada às rupturas de interface, de conteúdo, assim como rupturas originadas de suas restrições linguísticas na LP (sua L2).

O MISI foi aplicado para avaliar aspectos de comunicabilidade da interface em LP para alunos surdos, no AVA da UFMG Virtual, customizado a partir do Moodle. O processo da aplicação do MISI neste trabalho seguiu três fases distintas: preparação 
(delineamento do escopo, recrutamento dos participantes, preparação para a coleta de dados), coleta de dados (inspeção do sistema com os usuários indiretos, mediada pela entrevista conduzida por especialistas) e análise (preparação, análise dos dados e, por fim, a interpretação dos dados). Para sua aplicação, utilizamos o escopo da disciplina de "Fundamentos de Libras on-line", realizando algumas adaptações para o teste (Figura 1). O intuito foi avaliar a interface principal da UFMG Virtual e o layout da disciplina. Essa disciplina é ofertada, na modalidade à distância na UFMG Virtual, pela Faculdade de Letras da UFMG como disciplina obrigatória nas licenciaturas, Fonoaudiologia e Pedagogia e como disciplina eletiva em todos os cursos da UFMG.

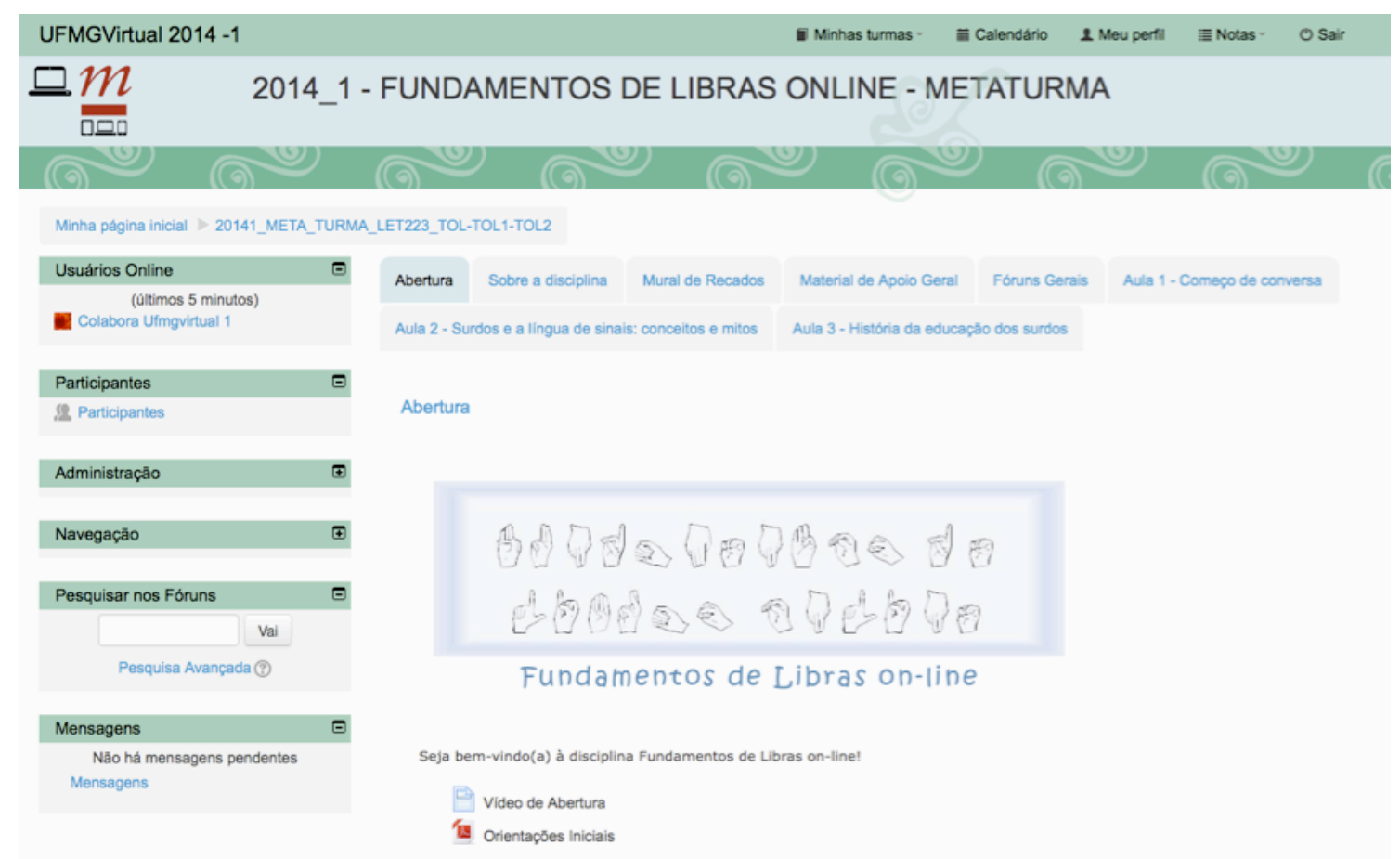

Figura 1: Tela principal da disciplina de Fundamentos de Libras

$\mathrm{Na}$ definição dos cenários, buscamos explorar os recursos mais utilizados nas aulas de Fundamentos de Libras on-line, destacando-se o uso dos fóruns de discussão, envio de tarefas e questionários. Optamos pelo uso dos fóruns e tarefas por serem dois recursos importantes e menos complexos para uso por alunos que nunca tiveram contato com o Moodle.

A avaliação teve a participação de duas avaliadoras, especialistas em IHC e EngSem, sendo que uma delas tinha experiência em pesquisa com alunos surdos e a outra já aplicava o MISI pela terceira vez. Como a pesquisa envolveu participantes surdos, a atuação da intérprete de Libras foi essencial para possibilitar a comunicação entre avaliadoras e os alunos surdos. Participaram desta avaliação duas profissionais tradutoras e intérpretes do par linguístico Língua Portuguesa/Língua Brasileira de Sinais (Libras), ambas com certificado de proficiência em Libras.

Para o recrutamento dos participantes, alunos surdos, utilizamos a estratégia do perfil de alta definição que consiste em maximizar a homogeneidade de uma amostra a partir de critérios relevantes. Delineamos um perfil que englobava aspectos gerais comuns a pessoas ouvintes (conhecimento de LP, nível de escolaridade, conhecimento 
de línguas, nível de conhecimento do Moodle, nível de conhecimento da tecnologia, perfil socioeconômico e faixa etária) e aspectos peculiares relacionados à LP e LS utilizada pelos surdos (nível de surdez e período do diagnóstico da surdez, nível de oralidade, comunicação em Libras e período em que aprendeu Libras). O quadro 1 apresenta um resumo do perfil com foco nos aspectos da surdez.

Quadro 1 - Perfil dos alunos surdos participantes do MISI

\begin{tabular}{|c|c|c|c|c|}
\hline Critério/Perfil & Participante 1 & Participante 2 & Participante 3 & Participante 4 \\
\hline $\begin{array}{l}\text { Faixa etária: serem } \\
\text { jovens e adultos com faixa } \\
\text { etária entre } 20 \text { a } 45 \text { anos }\end{array}$ & 18 a 25 anos & 41 a 45 anos & 26 a 30 anos & 18 a 25 anos \\
\hline $\begin{array}{l}\text { Nível de surdez e } \\
\text { época/período do } \\
\text { diagnóstico da surdez: } \\
\text { alunos surdos profundos, } \\
\text { pré-linguais, sem implante } \\
\text { coclear }\end{array}$ & $\begin{array}{l}\text { profundo } \\
\text { pré-lingual } \\
\text { (nasceu surdo) } \\
\text { sem implante } \\
\text { coclear }\end{array}$ & $\begin{array}{l}\text { severo } \\
\text { pré-lingual } \\
\text { (nasceu surdo) } \\
\text { sem implante } \\
\text { coclear }\end{array}$ & $\begin{array}{l}\text { profundo } \\
\text { pré-lingual } \\
\text { (surdo antes } \\
\text { dos } 2 \text { anos) } \\
\text { sem implante } \\
\text { coclear }\end{array}$ & $\begin{array}{l}\text { profundo } \\
\text { pré-lingual } \\
\text { (nasceu surdo) } \\
\text { sem implante } \\
\text { coclear }\end{array}$ \\
\hline $\begin{array}{l}\text { Nível de oralidade: } \\
\text { surdos oralizados ou não; }\end{array}$ & $\begin{array}{l}\text { Oralizado } \\
\text { (aprendeu a } \\
\text { falar) } \\
\text { Faz leitura } \\
\text { labial }\end{array}$ & $\begin{array}{l}\text { Oralizado } \\
\text { (aprendeu a } \\
\text { falar) } \\
\text { Faz leitura } \\
\text { labial }\end{array}$ & $\begin{array}{l}\text { Não oralizado } \\
\text { Não faz leitura } \\
\text { labial }\end{array}$ & $\begin{array}{l}\text { Não oralizado } \\
\text { Não faz leitura } \\
\text { labial }\end{array}$ \\
\hline $\begin{array}{l}\text { Comunicação em } \\
\text { Libras: usuários de Libras } \\
\text { com proeficiência } \\
\text { intermediária }\end{array}$ & $\begin{array}{l}\text { Sim } \\
\text { Não tem } \\
\text { preferência na } \\
\text { forma de } \\
\text { comunicacão } \\
\text { (Libras, LP ou } \\
\text { leitura labial) }\end{array}$ & $\begin{array}{l}\text { Sim } \\
\text { Não tem } \\
\text { preferência na } \\
\text { forma de } \\
\text { comunicacão } \\
\text { (Libras, LP ou } \\
\text { leitura labial) }\end{array}$ & $\begin{array}{l}\text { Sim } \\
\text { Tem preferência } \\
\text { por se } \\
\text { comunicar em } \\
\text { Libras }\end{array}$ & $\begin{array}{l}\text { Sim } \\
\text { Tem preferência } \\
\text { por se } \\
\text { comunicar em } \\
\text { Libras }\end{array}$ \\
\hline $\begin{array}{l}\text { Período em que } \\
\text { aprendeu Libras: } \\
\text { preferencialmente, grupo } \\
\text { de surdos profundos, } \\
\text { pré-linguais, que } \\
\text { aprenderam Libras na } \\
\text { adolescência }\end{array}$ & $\begin{array}{l}\text { Antes dos } 5 \\
\text { anos de idade, } \\
\text { na escola }\end{array}$ & $\begin{array}{l}\text { Depois dos } 7 \\
\text { anos de idade } \\
\text { (aprox. } 14 \\
\text { anos), com } \\
\text { amigos }\end{array}$ & $\begin{array}{l}\text { Antes dos } 5 \\
\text { anos de idade, } \\
\text { na escola }\end{array}$ & $\begin{array}{l}\text { Depois dos } 7 \\
\text { anos de idade } \\
\text { (aprox. } 11 \\
\text { anos), na escola }\end{array}$ \\
\hline
\end{tabular}

Em relação ao nível de formação dos participantes, apenas um deles estava matriculado em curso superior e os demais haviam concluído o ensino técnico. Todos tinham conhecimento de LP para leitura e escrita, possuíam experiência de nível básico/intermediário no uso de tecnologias. Um dos participantes, o P1, conhecia o Moodle, mas nunca tinha feito uso desse ambiente para fins de aprendizagem. Já o P4 tinha feito uso do Moodle somente para acesso a arquivos PDF e os demais não o conheciam. Com isso, foi possível evitar que o conhecimento prévio dos participantes em relação ao sistema, impactasse nos resultados.

Pelo fato do MISI ser um método qualitativo, foram realizadas quatro avaliações do MISI com alunos surdos, sendo um teste piloto e quatro avaliações. O teste piloto foi importante para validar o material de teste gerado [Nicolaci-Da-Costa et al., 2004]. Todas as avaliações foram realizadas em ambiente controlado (e.g. laboratório de testes). Durante a realização dos testes com alunos surdos, a comunicação oral e o conteúdo do material impresso foram traduzidos de LP para Libras pela intérprete. As dúvidas dos surdos foram traduzidas de Libras para LP. 


\section{Resultados}

A aplicação do MISI na interface da disciplina Fundamentos de Libras on-line permitiu identificar categorias relevantes a serem consideradas no projeto de um AVA, a fim de promover acessibilidade para alunos surdos, assim como identificar as principais rupturas na UFMG Virtual.

Em nosso estudo, as categorias envolveram o Moodle (num escopo mais geral), a UFMG Virtual (Moodle customizado) e a disciplina de Fundamentos de Libras on-line (um escopo dentro da UFMG Virtual). Estas categorias, sob o ponto de vista dos alunos surdos, foram: (1) Uso de vídeos em Libras: todos os participantes consideraram de grande relevância apresentar vídeos em Libras de forma a promover uma melhor compreensão do conteúdo pelos alunos surdos; (2) Nomenclatura dos rótulos: alguns rótulos em LP presentes em botões, menus e links na interface não foram bem compreendidos (i.e. fora da nomenclatura padrão, palavras desconhecidas em LP ou em língua inglesa); (3) Layout das telas: a posição e tamanho dos elementos presentes na interface também interferiu na comunicação (i.e. falta de hierarquização ou pouco destaque de elementos principais); (4) Sistema de ajuda: a UFMG Virtual e o Moodle oferecem pouco conteúdo de ajuda para os alunos, o que foi considerado essencial para contribuir com uma interação adequada por parte deles diante de dúvidas de uso e (5) Tamanho dos textos em LP: a utilização de textos simples e objetivos em LP, foi uma característica destacada que contribui para a sua compreensão por alunos surdos. Estas categorias estão relacionadas aos principais problemas apontados por eles durante a inspeção.

Apresentamos, a seguir, a interpretação das principais rupturas encontradas na interface da UFMG Virtual, também aplicadas no contexto do Moodle, durante a aplicação do MISI. A tela principal da interface da UFMG Virtual e da disciplina de Fundamentos de Libras on-line está apresentada na Figura 1. Ela envolve um menu superior (Minhas Turmas, Calendário, Meu Perfil, Notas e Sair) e blocos laterais (Usuários on-line, Participantes, Administração, Navegação, Pesquisar nos fóruns e Mensagens). Por questões de limitação de espaço, apresentamos os resultados do MISI para um bloco lateral de grande relevância "Administração" e ao uso do fórum (como uma das atividades propostas) ${ }^{1}$. Para cada um desses elementos do design, identificamos o tipo de ruptura (interface, conteúdo ou linguística) e propusemos sugestões de melhorias. Durante a interação, os surdos expressavam suas dúvidas, o que permitiu diferenciar a origem da ruptura. Em outras situações o surdo era questionado com a finalidade de identificar a origem da ruptura.

\subsection{Bloco Administração}

Neste bloco, identificamos rupturas relacionadas à interface e aos aspectos linguísticos. As categorias do MISI identificadas na interação, neste bloco, foram: (1) Uso de vídeos em Libras, (2) Nomenclatura dos rótulos e (4) Sistema de ajuda.

Os participantes não compreenderam adequadamente a terminologia associada ao nome do bloco, o que interpretamos como uma ruptura de origem linguística. Cada participante associou a elementos distintos (i.e. notas, relatórios, projetos, etc.). $\mathrm{Na}$

\footnotetext{
1 Os demais resultados podem ser consultados no relatório do MISI, disponível em http://www.leticiacapelao.com/curriculo_prof_pesquisa_public.html
} 
seção Meu Perfil, P3, por exemplo, ressaltou a ausência de Libras como valor para o campo Idioma preferido e, na parte de descrição, sugeriu a inclusão de vídeos em Libras como descrição do perfil para alunos surdos. O conteúdo do tooltip (dicas de uso exibidas em janelas que surgem ao passar o mouse sobre uma imagem ou texto na

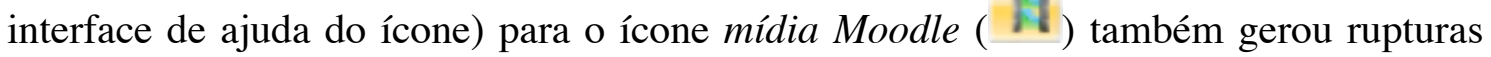
em sua compreensão.

A partir dos problemas observados pelos surdos durante a inspeção com o MISI, apresentamos sugestões de melhoria, que são listados de Adm1 e Adm5. Adm1. Utilizar a terminologia Meus dados se referindo às suas notas, perfil e relatórios. Essa terminologia atende ao contexto de uso do aluno (usuário direto e de grande relevância no uso do AVA), assim como ao contexto de uso de professores, designers instrucionais, tutores e monitores. Adm2. Permitir a inclusão de vídeo em Libras com tamanho máximo de upload, como formato de descrição do perfil, específico para atender acessibilidade para alunos surdos. Este campo é restrito para pessoas surdas, como um recurso de acessibilidade. Adm3. Alterar o conteúdo do tooltip do ícone mídia Moodle de Insira mídia Moodle para Inserir som, vídeo ou applet. Adm4. Incluir Libras como um dos valores do campo Idioma preferido na opção de modificação do perfil. Caso não seja possível (por estar relacionado ao idioma de tradução do sistema) incluir informação de ajuda para esclarecer sobre os valores deste campo. Adm5. Incluir informação de ajuda a fim de possibilitar o uso de todos os seus recursos e compreensão das informações exibidas na tela, de grande relevância para o aluno. Esse recurso de ajuda pode ser incluído através de uma breve descrição na própria tela, abaixo do nome do rótulo, através de tooltips ou através de janelas pop-ups que são exibidas a partir de um botão de ajuda (por exemplo, “?”).

\subsection{Uso do fórum}

Durante a interação com o fórum, observamos rupturas na interface da tela de feedback desse recurso e no campo de postagem da resposta ao fórum. Identificamos rupturas relacionadas à interface, bem como rupturas linguísticas. Todas as categorias do MISI foram identificadas na interação do fórum (1, 2, 3, 4 e 5).

$\mathrm{Na}$ tela de feedback, após enviar a mensagem ao fórum, o sistema exibiu uma mensagem por um período de 3 segundos, impossibilitando a leitura. Além disso, o texto estava em azul (cor usada para links) com uma tarja em cor de fundo azul, dificultando a leitura. Já no campo de postagem da resposta, consideramos que houve uma ênfase visual na interface para o recurso do Anexo pela presença de uma imagem em cor azul (uma seta), pois os participantes tiveram sua atenção voltada para esta parte da interface e não compreenderam onde deveriam postar a resposta.

Para evitar problemas na comunicação e um foco na postagem da mensagem, apresentamos as seguintes sugestões de melhorias, apresentados como For1 a For8. For1. Alterar o nome do rótulo Mensagem para Sua resposta. For2. Incluir uma mensagem de texto dentro da caixa de mensagem. Por exemplo, Escreva aqui a sua resposta para o fórum. For3. Alterar a combinação de cores da mensagem exibida do feedback do fórum após a postagem para cor de texto padrão e sem link e a cor de tarja em cinza, ou azul/verde que são cores usadas na interface da UFMG Virtual. For4. Solicitar ao usuário clicar no botão para prosseguir, em vez de prosseguir automaticamente depois de curto período de tempo. Outro recurso é criar um feedback 
persistente em uma região da tela, isto é, a mensagem é exibida até que o usuário interaja novamente com o sistema. For5. Utilizar textos mais curtos e simples nos enunciados das atividades. For6. Incluir os enunciados das atividades com vídeos em Libras a fim de que os alunos surdos possam realizar a sua leitura sem a necessidade de solicitar ajuda. For7. Incluir legenda em LP para os vídeos em Libras ou apresentar tradução textual abaixo do vídeo, em virtude das diferenças entre sinais para uma mesma palavra e pelas diferentes competências linguísticas na Libras entre os surdos. For8. Incluir informação de ajuda para uso dos fóruns a fim de possibilitar o uso dos seus recursos e compreensão das informações exibidas na tela. Esse recurso de ajuda pode ser incluído através de uma breve descrição na própria tela abaixo do nome do rótulo, através de tooltips ou através de janelas pop-ups que são exibidas a partir de um botão de ajuda (por exemplo, “?”).

\section{Conclusões e Trabalhos Futuros}

Uma vez que toda proposta de EaD é projetada para atingir um objetivo principal de ensino-aprendizagem, é essencial que o aluno compreenda os elementos do design que contribuem para a comunicação na interface. Nosso objetivo foi investigar as possibilidades de interação dos surdos com a LP de várias formas e as rupturas de comunicação identificadas no Moodle da UFMG Virtual, usando como cenário a disciplina Fundamentos de Libras. Neste sentido, trazemos contribuições para duas áreas distintas: informática e educação e IHC. Para a área de informática e educação contribui por permitir apreciar a qualidade de comunicação de um AVA, em LP, para surdos. Assim, tentamos agregar mais esforços para a integração e a permanência dos surdos no ensino superior, explorando os recursos da EaD. Para a área de IHC, por outro lado, realçamos nossa contribuição pela aplicação do MISI, um método recentemente proposto, buscando sua apreciação em um ambiente educacional, conforme sua proposta original, com um novo perfil de usuários: os surdos.

A aplicação do MISI trouxe diferentes contribuições: (1) a descrição do uso do MISI para avaliação com alunos surdos auxilia na pesquisa em diferentes contextos (considerando acessibilidade); (2) a apresentação de decisões sobre como aplicar o MISI com pessoas surdas é útil para quem quer realizar testes com este perfil de usuários; (3) o foco no usuário surdo contribuiu para a geração de conhecimento sobre as dificuldades desses usuários, assim como a adequação das tecnologias ao uso por eles. Assim, a descrição dos problemas apresentados pode evitar que eles ocorram em outros sistemas; (4) a identificação de problemas e apresentação de sugestões de melhorias para a interface da UFMG Virtual e da disciplina de Fundamentos de Libras podem contribuir para uma melhor comunicabilidade e, consequentemente, acessibilidade dos surdos ao sistema, assim como ouvintes; (5) os problemas encontrados foram relevantes sobre o uso do sistema e serão comunicados aos responsáveis, podendo gerar melhorias na sua qualidade tanto para surdos, quanto para ouvintes; (6) o MISI permitiu identificar as rupturas de interface, linguística (relacionadas à LP ou Libras) e de conteúdo (disponibilizado pelo professor), diferenciando problemas relacionados a cada um desses três aspectos, resultando numa intermediação positiva e fundamental para os surdos e avaliadores.

Considerando a importância da Libras como L1 para os surdos, a sua relevância destacada pelos alunos durante a aplicação do MISI e a viabilidade do uso de avatares (intérpretes virtuais) como um recurso de acessibilidade para os surdos, pretendemos 
incluir um avatar de Libras (i.e. HandTalk) em trabalhos futuros na UFMG Virtual e, então, avaliar sua comunicabilidade com alunos surdos fazendo uso do MISI. Os avatares têm-se mostrado como alternativas convenientes ao uso de vídeos devido às seguintes vantagens: facilidade de uso, aplicabilidade, baixo custo e manutenção. Logo, sua validação em um contexto de aprendizagem, com alunos surdos, visa contribuir com a acessibilidade dos surdos na $\mathrm{EaD}$, complementando e ampliando estudos já realizados.

\section{Referências}

Capelão, L. et al. (2011) Avaliação de comunicabilidade do Moodle para usuários surdos e ouvintes. In: IHC2010 - Anais Estendidos Competição, Competição, 2011, Porto de Galinhas. CLIHC'2011: IHC.

Capelão, L. (2015) Recomendações para cursos on-line em língua portuguesa com foco na integração de alunos surdos. 2014. xxx f. Tese (Doutorado em Linguística Aplicada). Faculdade de Letras, UFMG. Belo Horizonte. (no prelo)

Capterra.com (2014). Big Changes in the Top 20 Most Popular LMS Rankings. Disponível em <http://blog.capterra.com/big-changes-most-popular-lms-rankings>. Acesso em 25 mai. 2015.

De Oliveira, E. R. (2010) Investigação sobre a aplicabilidade dos métodos de avaliação de comunicabilidade ao domínio educacional. 206 f. Dissertação (Mestrado em Ciência da Computação) - DCC, UFMG. Belo Horizonte.

De Souza, C. S. (2005) The Semiotic Engineering of Human-Computer Interaction. MIT Press, Cambridge MA, U.K.

Flor, C. et al. Recomendações para o design de interfaces web acessíveis ao público surdo. In: VII World Congress on Communication and Arts. Portugal: Vila Real, 2014. p. 50-54.

Nicolaci-Da-Costa, A. M.; Leitão, C. F.; Romão-Dias, D. (2004) Como conhecer usuários através do Método de Explicitação do Discurso Subjacente (MEDS). Anais do IHC 2004, Curitiba.

Pivetta, E. M. et al. (2013) Contribuições para o design de interface de um Ambiente Virtual de Ensino Aprendizagem acessível a surdos. Revista Brasileira de Design da Informação, v. 10, n. 2, p. 193-206.

Prates, R. O. (2004) A Engenharia Semiótica para o domínio educacional. In: Workshop de Design e Avaliação de Interfaces para Ambientes Educacionais. Curitiba: IHC.

Preece, J.; Rogers, Y.; Sharp, H. (2011) Interaction Design: Beyond Human-Computer Interaction. John Wiley \& Sons; 3rd Edition.

Rocha, D. F. S et al. (2014) Uma Revisão Sistemática sobre a Educação do Surdo em Ambientes Virtuais Educacionais. In: XXV Simpósio Brasileiro de Informática na Educação (SBIE). 